\title{
Linear Eyring Plots Conceal a Change in the Rate-Limiting Step in an Enzyme Reaction
}

\author{
Teresa F. G. Machado, ${ }^{\dagger}$ Tracey M. Gloster, ${ }^{\ddagger}$ and Rafael G. da Silva* ${ }^{*} \neq 0$ \\ ${ }^{\dagger}$ School of Chemistry and ${ }^{\ddagger}$ School of Biology, Biomedical Sciences Research Complex, University of St Andrews, St Andrews, Fife \\ KY16 9ST, United Kingdom
}

Supporting Information

ABSTRACT: The temperature dependence of psychrophilic and mesophilic (R)-3-hydroxybutyrate dehydrogenase steady-state rates yields nonlinear and linear Eyring plots, respectively. Solvent viscosity effects and multipleand single-turnover pre-steady-state kinetics demonstrate that while product release is rate-limiting at high temperatures for the psychrophilic enzyme, either interconversion between enzyme-substrate and enzyme-product complexes or a step prior to it limits the rate at low temperatures. Unexpectedly, a similar change in the rate-limiting step is observed with the mesophilic enzyme, where a step prior to chemistry becomes ratelimiting at low temperatures. This observation may have implications for past and future interpretations of temperature-rate profiles.

$\mathrm{T}$ emperature-rate profiles are powerful tools for gaining insight into thermodynamic activation parameters of chemical reactions. ${ }^{1}$ In enzyme-catalyzed reactions, analysis of the temperature-dependent behavior of rate constants is widely used to uncover catalytic properties among psychrophilic, mesophilic, and thermophilic enzymes ${ }^{2}$ and to probe protein dynamics and hydrogen tunneling ${ }^{3}$ and even allosteric regulation., ${ }^{4,5}$ Plotting $\ln (k / T)$ versus $1 / T$ (Eyring plot) often results in a straight line, and fitting data to eq 1 yields the activation enthalpy and entropy, assuming they are constant over the temperature range and recrossing is negligible., ${ }^{1,6,7}$ However, nonlinear Eyring plots have been reported, ${ }^{8}$ and a recent and elegant hypothesis for interpreting them invokes a role for activation heat capacity in enzyme catalysis upon fitting data to eq $2 .{ }^{9,10}$ In eqs 1 and $2, k$ is the rate constant; $k_{\mathrm{B}}, h$, and $R$ are the Boltzmann, Planck, and gas constants, respectively; $\Delta H^{\ddagger}$ and $\Delta S^{\ddagger}$ are the activation enthalpy and entropy, respectively; $T$ is the temperature; $T_{0}$ is a reference temperature; $\Delta H^{\ddagger} T_{0}$ and $\Delta S^{\ddagger} T_{0}$ are $\Delta H^{\ddagger}$ and $\Delta S^{\ddagger}$ at $T_{0}$, respectively; and $\Delta C_{p}^{\ddagger}$ is the activation heat capacity.

$$
\begin{gathered}
\ln \frac{k}{T}=\ln \frac{k_{\mathrm{B}}}{h}-\frac{\Delta H^{\ddagger}}{R T}+\frac{\Delta S^{\ddagger}}{R} \\
\ln \frac{k}{T}=\ln \frac{k_{\mathrm{B}}}{h}-\frac{\Delta H^{\ddagger}{ }_{T_{0}}+C_{p}^{\ddagger}\left(T-T_{0}\right)}{R T} \\
+\frac{\Delta S_{T_{0}}^{\ddagger}+C_{p}^{\ddagger} \ln \left(T / T_{0}\right)}{R}
\end{gathered}
$$

Nonlinear Eyring plots without protein denaturation could also arise from a switch in the rate-limiting step at a given temperature, which must be tested. ${ }^{2,8}$ Conversely, linear Eyring plots are often assumed to reflect the same rate-limiting step at all temperatures. ${ }^{2,6,9,11,12}$ Here we show that linear Eyring plots may accommodate a change in the rate-limiting step.

We cloned and expressed the bacterial $(R)$-3-hydroxybutyrate dehydrogenase $(\mathrm{HBDH})$-encoding gene from mesophilic Acinetobacter baumannii ${ }^{13}$ and from psychrophilic Psychrobacter $\operatorname{arcticus}^{14}$ and purified the recombinant mesophilic (AbHBDH) and psychrophilic $(\mathrm{PaHBDH})$ enzymes (Figure S1). HBDH (EC 1.1.1.30) catalyzes the $\mathrm{NADH}$-dependent reduction of acetoacetate to (R)-3-hydroxybutyrate, with 3-oxovalerate being an alternative substrate turned over more slowly by the enzyme (Scheme S1). ${ }^{15}$

HBDH is part of the short-chain dehydrogenase/reductase (SDR) superfamily, one of the largest known protein groups. ${ }^{16}$ SDR proteins share a low level of sequence identity but highly conserved three-dimensional architecture and catalytic residues. ${ }^{17} \mathrm{HBDH}$ has applications in asymmetric synthesis of precursors of pharmaceuticals and monomeric constituents of biodegradable polyhydroxyalkanoates. ${ }^{18,19}$

We measured saturation curves for $\mathrm{AbHBDH}$ and $\mathrm{PaHBDH}$ at various temperatures $(283-330 \mathrm{~K}$ for $A b \mathrm{HBDH}$ and $283-$ $318 \mathrm{~K}$ for $\mathrm{PaHBDH}$ ) (Figure $\mathrm{S} 2$ and Tables $\mathrm{S} 1-\mathrm{S} 4$ ). Differential scanning fluorimetry demonstrated no denaturation occurs in this temperature range (Figure $\mathrm{S} 3$ and Table S5). Apparent steady-state catalytic constants $\left(k_{\text {cat }}\right)$ were used to construct Eyring plots for each enzyme (Figure 1). Fitting all data with both eqs 1 and 2 resulted in best-fit lines that are linear for $\mathrm{A} b \mathrm{HBDH}$ but nonlinear for $\mathrm{PaHBDH}$. Accordingly, a

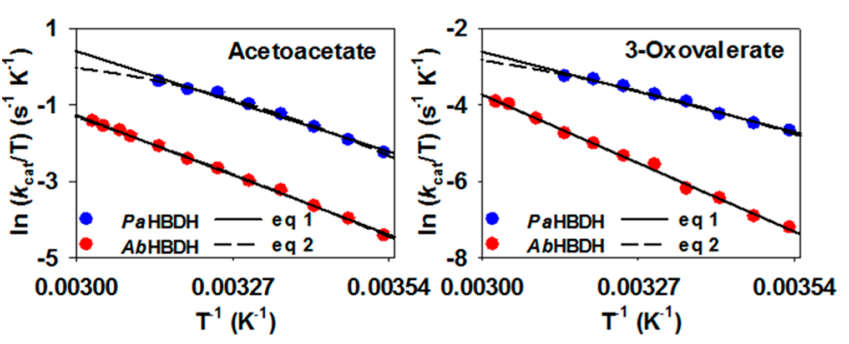

Figure 1. Eyring plots for $\mathrm{PaHBDH}$ and $\mathrm{AbHBDH}$ with acetoacetate and 3-oxovalerate as substrates.

Received: October 15, 2018

Revised: November 12, 2018

Published: November 26, 2018 
significant negative $\Delta C_{p}^{\ddagger}$ is predicted for the psychrophilic enzyme but not for the mesophilic one (Table S6).

A negative $\Delta C_{p}^{\ddagger}$ for $k_{\text {cat }}$ could suggest a decrease in the number of vibrational modes as the Michaelis complex progresses toward the transition state..$^{9,11,12,20}$ However, enzymatic rates often contain contributions from several steps, and a negative $\Delta C_{p}^{\ddagger}$ can report on vibrational modes associated with chemical ${ }^{10,20}$ and physical steps. ${ }^{2}$

To test if diffusional steps limit the steady-state rate in $\mathrm{PaHBDH}$ and $\mathrm{AbHBDH}$ reactions at $298 \mathrm{~K}$, we determined solvent viscosity effects by measuring saturation curves at different concentrations of glycerol (Figure S4 and Tables S7S10), and plots of $k_{\text {cat }}$ ratios versus relative viscosity ${ }^{21}$ are shown in Figure 2. Fitting data to eq S5 yielded slopes of 0.43
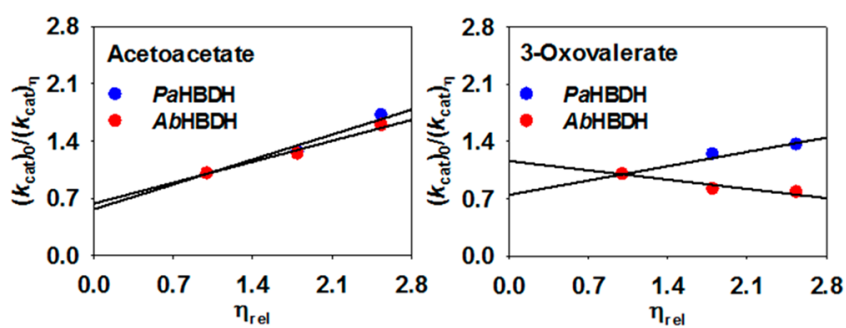

Figure 2. Solvent viscosity effects on $k_{\text {cat }}$ for $\mathrm{PaHBDH}$ and $\mathrm{AbHBDH}$ with acetoacetate and 3-oxovalerate as substrates. Lines are fits of data to eq S5.

\pm 0.04 and $0.36 \pm 0.02$ for $P a \mathrm{HBDH}$ and $A b \mathrm{HBDH}$, respectively, indicating product release is significantly ratelimiting with acetoacetate as the substrate. ${ }^{21,22}$ With 3oxovalerate as the substrate, slopes decrease to $0.25 \pm 0.02$ for $\mathrm{PaHBDH}$, consistent with only a modest product release contribution to the rate-limiting step, and $-0.16 \pm 0.02$ for $A b \mathrm{HBDH}$, showing no contribution from diffusional steps to $k_{\text {cat }}$. Diffusional steps from substrate binding do not limit reaction rates for either enzyme (Figure S4), and 5\% PEG8000 as a macroviscogen had no effect on saturation curves (Figure S5), pointing to the effects of glycerol resulting from an increase in solvent microviscosity. ${ }^{21,23}$

The solvent viscosity effects on $k_{\text {cat }}$ suggest that the curvatures in the Eyring plots and the consequent negative $\Delta C_{p}^{\ddagger}$ calculated for $\mathrm{PaHBDH}$ may report on distinct steps when acetoacetate and 3-oxovalerate are used as substrates. To probe further the nature of the rate-limiting step for this enzyme, we employed multiple-turnover pre-steady-state kinetics to characterize the approach to the steady state ${ }^{24}$ at 283, 298, and $318 \mathrm{~K}$ (Figure 3A). No burst in substrate consumption is observed with 3-oxovalerate as the substrate. These results support the conclusion from solvent viscosity effects at $298 \mathrm{~K}$ that $k_{\text {cat }}$ is not limited by the product release rate with 3-oxovalerate as the substrate.

When acetoacetate is used as the substrate, even though a burst in substrate depletion at 298 and $318 \mathrm{~K}$ cannot be directly observed, it can be inferred ${ }^{25}$ (Figure 3A). The concentration of co-substrate $\mathrm{NADH}$ at time zero in the presence of $\mathrm{PaHBDH}$ and acetoacetate is significantly offset from its value in the control without acetoacetate (Figure S6), suggesting at least one turnover has happened within the 0.9 $\mathrm{ms}$ dead time of the stopped-flow spectrophotometer. This suggests a step after chemistry, likely product release, is ratelimiting at 298 and $318 \mathrm{~K}$, in agreement with the significant solvent viscosity effect on $k_{\text {cat }}$ at $298 \mathrm{~K}$. At $283 \mathrm{~K}$, however,
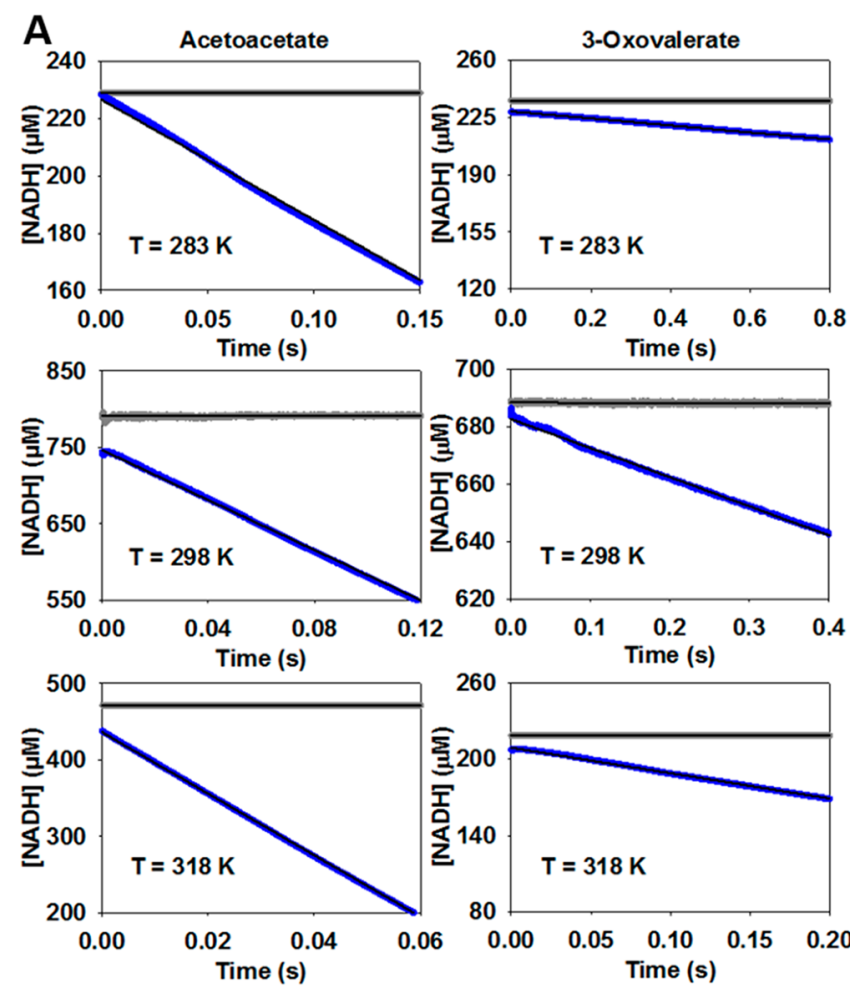

No acetoacetate or 3-oxovalerate $\quad 25 \mu \mathrm{M}$ PaHBDH
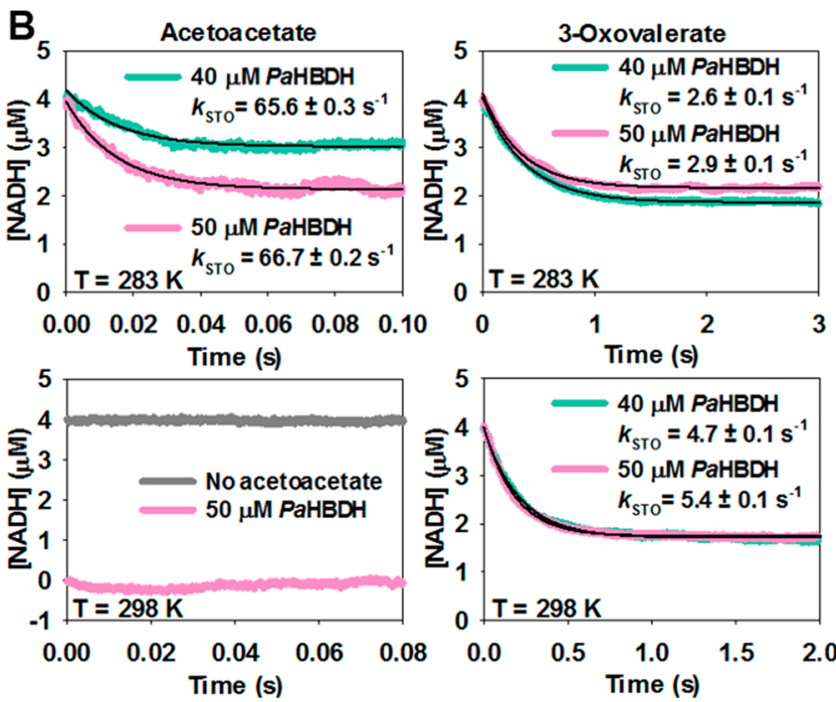

Figure 3. Rapid kinetics for $\mathrm{PaHBDH}$ with acetoacetate and 3oxovalerate as substrates. (A) Multiple-turnover pre-steady-state kinetics. Black lines are fits of data to eq S3. (B) Single-turnover pre-steady-state kinetics. Black lines are fits of data to eq S4.

there is no burst (Figure 3A and Figure S6), indicating a change in the rate-limiting step at low temperatures.

The hypotheses regarding rate-limiting steps in the $\mathrm{PaHBDH}$ reaction at different temperatures were tested further by single-turnover pre-steady-state kinetics at 283 and $298 \mathrm{~K}$ with $\mathrm{NADH}$ as the limiting reagent. $\mathrm{PaHBDH}$ singleturnover rate constants $\left(k_{\mathrm{STO}}\right)$ were independent of enzyme concentration (Figure 3B), demonstrating $\mathrm{NADH}$ is saturated with $\mathrm{PaHBDH}$ and $k_{\mathrm{STO}}$ is unimolecular. ${ }^{24}$ With 3-oxovalerate, $k_{\text {STO }}$ and $k_{\text {cat }}$ values are similar at the same temperatures (Table S11). Along with the absence of a pre-steady-state burst and a negligible solvent viscosity effect on $k_{\text {cat }}$ with this substrate, this 
result indicates that interconversion between $\mathrm{PaHBDH}$ $\mathrm{NADH}$-3-oxovalerate and $\mathrm{PaHBDH}-\mathrm{NAD}^{+}$-3-hydroxyvalerate is rate-limiting. With acetoacetate as the substrate, if the hypothesis emerging from multiple-turnover pre-steady-state kinetics and solvent viscosity effects holds, $k_{\text {STO }}$ should be measurable at $283 \mathrm{~K}$ but should not be possible to monitor at $298 \mathrm{~K}$ as it takes place within the dead time of the instrument. This is exactly what is observed (Figure $3 \mathrm{~B}$ and Table S11), with $k_{\text {STO }}$ being too fast to be detected at $298 \mathrm{~K}$ although $\mathrm{NADH}$ is consumed. At $283 \mathrm{~K}, k_{\text {STO }}$ is 2.2 -fold higher than $k_{\text {cat }}$ which, combined with the absence of a burst, indicates a step prior to acetoacetate reduction is rate-limiting at this temperature.

Whereas there is precedent for a change in the rate-limiting step in nonlinear Eyring plots of enzyme reactions, ${ }^{26} \mathrm{PaHBDH}$ Eyring plots are linear in the range of 283-308 K (Figure S7), and the switch in the rate-limiting step occurs between 283 and $298 \mathrm{~K}$. This indicates a temperature-dependent shift in the rate-limiting step giving rise to a linear Eyring plot.

To assess whether this unusual behavior is displayed by the mesophilic enzyme, we carried out rapid kinetics experiments with $A b \mathrm{HBDH}$, whose Eyring plots are linear throughout the experimental temperature range. Remarkably, multiple-turnover pre-steady-state kinetics at 283, 298, and $328 \mathrm{~K}$ (Figure 4A) show a change in the rate-limiting step with both substrates. A burst in substrate consumption at 298 and $328 \mathrm{~K}$ with acetoacetate and at $328 \mathrm{~K}$ with 3 -oxovalerate is too fast to be directly observed but is clearly inferred ${ }^{25}$ from the offset in $\mathrm{NADH}$ concentration at time zero in the presence and absence of acetoacetate or 3-oxovalerate (Figure S6), indicating the first turnover occurs within the dead time of the stopped-flow spectrophotometer. Conversely, there is no burst at $283 \mathrm{~K}$ with either substrate or at $298 \mathrm{~K}$ with 3-oxovalerate (Figure 4A and Figure S6). The results at $298 \mathrm{~K}$ are in strict agreement with the conclusions drawn from solvent viscosity effects for both substrates.

As with $\mathrm{PaHBDH}$, we performed single-turnover pre-steadystate kinetics experiments at 283 and $298 \mathrm{~K}$, with NADH as the limiting reagent, to examine further the hypothesis of a change in the rate-limiting step in $A b \mathrm{HBDH}$ catalysis at different temperatures. $A b \mathrm{HBDH} k_{\text {STO }}$ values were independent of enzyme concentration (Figure 4B), demonstrating $\mathrm{NADH}$ is saturated with $\mathrm{AbHBDH}$ and $k_{\mathrm{STO}}$ is unimolecular. The $k_{\text {STO }}$ for acetoacetate reduction can be measured at $283 \mathrm{~K}$, but it is too fast to be monitored at $298 \mathrm{~K}$, even though $\mathrm{NADH}$ is consumed. This is in accordance with the results from rapid kinetics under multiple-turnover conditions. The $k_{\mathrm{STO}}$ for 3oxovalerate reduction can be measured at 283 and $298 \mathrm{~K}$, as predicted by the absence of a burst at these temperatures. The affinity between $A b \mathrm{HBDH}$ and its substrates decreases severely at high temperatures, which prevented single-turnover experiments at $328 \mathrm{~K}$ (the enzyme could not be concentrated enough to ensure sufficient binding to $\mathrm{NADH}$ ). Combined, these results demonstrate a shift in the rate-limiting step between 283 and $298 \mathrm{~K}$ for $A b \mathrm{HBDH}$-catalyzed reduction of acetoacetate and between 298 and $328 \mathrm{~K}$ for reduction of 3oxovalerate despite linear Eyring plots with both substrates.

The $k_{\mathrm{STO}} \mathrm{s}$ for $A b \mathrm{HBDH}$-catalyzed reduction of 3-oxovalerate are 10 - and 7 -fold higher than $k_{\text {cat }}$ at 283 and $298 \mathrm{~K}$, respectively, and the $k_{\text {STO }}$ for acetoacetate reduction at $283 \mathrm{~K}$ is 8-fold higher than the corresponding $k_{\text {cat }}$ (Table S12). Therefore, unlike the $\mathrm{PaHBDH}$ reaction, a step preceding chemistry limits the $A b \mathrm{HBDH}$ reaction rate with 3-oxovalerate
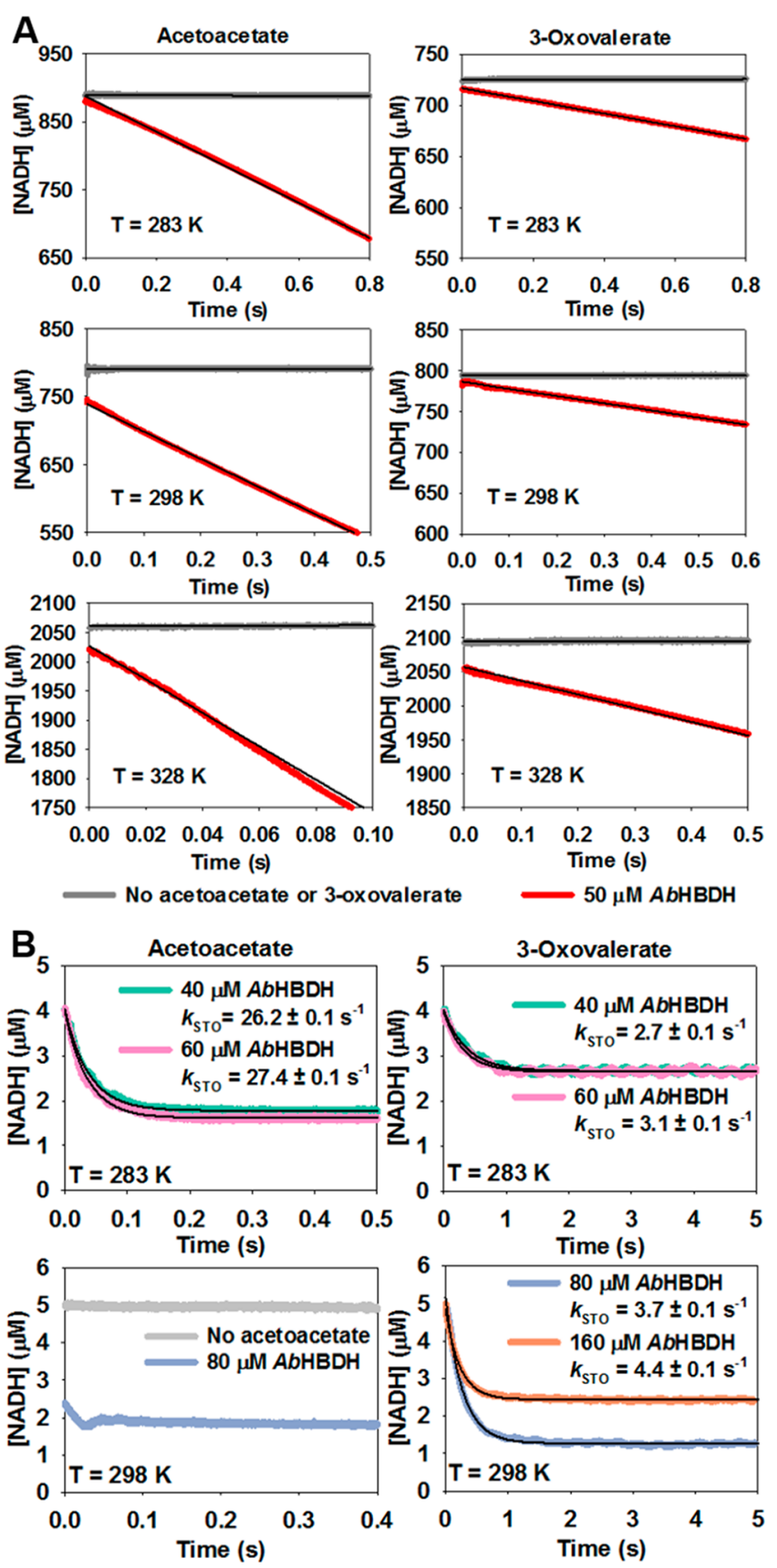

Figure 4. Rapid kinetics for $A b \mathrm{HBDH}$ with acetoacetate and 3oxovalerate as substrates. (A) Multiple-turnover pre-steady-state kinetics. Black lines are fits of data to eq S3. (B) Single-turnover pre-steady-state kinetics. Black lines are fits of data to eq S4.

at 283 and $298 \mathrm{~K}$ and with acetoacetate at $283 \mathrm{~K}$. Distinct ratelimiting steps between enzyme orthologues have been reported for other members of the SDR superfamily. ${ }^{27,28}$

Temperature-rate profiles are invaluable for the investigation of enzymatic mechanisms, encompassing probing quantum mechanical tunneling, ${ }^{3}$ enzyme dynamics, ${ }^{10,12}$ allostery, ${ }^{4,5}$ and calculation of thermodynamic parameters of activation. ${ }^{6,9-12}$ They are also instrumental for studying the evolution of modern enzymes from primitive catalysts that arose in a hot Earth. ${ }^{2,29,30}$

A temperature-dependent change in the rate-limiting step could produce a linear Eyring plot for $k_{\text {cat }}$ if the ratio of Eyring plot slopes for the microscopic rate constant(s) determining 
each step is relatively small. This scenario is demonstrated (Supporting Information) from simulated data (Figure S8) for a hypothetical two-step mechanism (Scheme S2) and may explain the results with $A b \mathrm{HBDH}$. This highlights the importance of confirming whether the same rate-limiting step holds throughout the experimental temperature range before further interpretation of both nonlinear and linear Eyring plots of macroscopic rate constants.

\section{ASSOCIATED CONTENT}

\section{S Supporting Information}

The Supporting Information is available free of charge on the ACS Publications website at DOI: 10.1021/acs.biochem.8b01099.

Materials and Methods, Schemes S1 and S2, Figures S1-S8, a discussion of simulated Eyring plots, and Tables S1-S12 (PDF)

\section{AUTHOR INFORMATION}

\section{Corresponding Author}

*E-mail: rgds@st-andrews.ac.uk.

ORCID $\odot$

Rafael G. da Silva: 0000-0002-1308-8190

\section{Funding}

This work was supported by the Engineering and Physical Sciences Research Council (EPSRC) (Grant EP/L016419/1) via a CRITICAT Centre for Doctoral Training studentship to T.F.G.M. T.M.G. is a Wellcome Trust Career Development Fellow.

\section{Notes}

The authors declare no competing financial interest.

\section{ACKNOWLEDGMENTS}

The authors thank Dr. Andrew Murkin for insightful discussions.

\section{ABBREVIATIONS}

HBDH, (R)-3-hydroxybutyrate dehydrogenase; SDR, shortchain dehydrogenase/reductase; $\Delta C_{p}^{\ddagger}$, activation heat capacity; $k_{\text {cat }}$, steady-state catalytic constant; $k_{\text {STO }}$, single-turnover rate constant.

\section{REFERENCES}

(1) Eyring, H. (1935) The activated complex in chemical reactions. J. Chem. Phys. 3, 107-115.

(2) Nguyen, V., Wilson, C., Hoemberger, M., Stiller, J. B., Agafonov, R. V., Kutter, S., English, J., Theobald, D. L., and Kern, D. (2017) Evolutionary drivers of thermoadaptation in enzyme catalysis. Science 355, 289-294.

(3) Kohen, A., Cannio, R., Bartolucci, S., Klinman, J. P., and Klinman, J. P. (1999) Enzyme dynamics and hydrogen tunnelling in a thermophilic alcohol dehydrogenase. Nature 399, 496-499.

(4) Lisi, G. P., Currier, A. A., and Loria, J. P. (2018) Glutamine hydrolysis by imidazole glycerol phosphate synthase displays temperature dependent allosteric activation. Front. Mol. Biosci. 5, 4.

(5) Saavedra, H. G., Wrabl, J. O., Anderson, J. A., Li, J., and Hilser, V. J. (2018) Dynamic allostery can drive cold adaptation in enzymes. Nature 558, 324-328.

(6) Winzor, D. J., and Jackson, C. M. (2006) Interpretation of the temperature dependence of equilibrium and rate constants. J. Mol. Recognit. 19, 389-407.

(7) Aqvist, J., Kazemi, M., Isaksen, G. V., and Brandsdal, B. O. (2017) Entropy and enzyme catalysis. Acc. Chem. Res. 50, 199-207.
(8) Truhlar, D. G., and Kohen, A. (2001) Convex arrhenius plots and their interpretation. Proc. Natl. Acad. Sci. U. S. A. 98, 848-851.

(9) Arcus, V. L., Prentice, E. J., Hobbs, J. K., Mulholland, A. J., Van der Kamp, M. W., Pudney, C. R., Parker, E. J., and Schipper, L. A. (2016) On the temperature dependence of enzyme-catalyzed rates. Biochemistry 55, 1681-1688.

(10) van der Kamp, M. W., Prentice, E. J., Kraakman, K. L., Connolly, M., Mulholland, A. J., and Arcus, V. L. (2018) Dynamical origins of heat capacity changes in enzyme-catalysed reactions. Nat. Commun. 9, 1177.

(11) Hobbs, J. K., Jiao, W., Easter, A. D., Parker, E. J., Schipper, L. A., and Arcus, V. L. (2013) Change in heat capacity for enzyme catalysis determines temperature dependence of enzyme catalyzed rates. ACS Chem. Biol. 8, 2388-2393.

(12) Arcus, V. L., and Pudney, C. R. (2015) Change in heat capacity accurately predicts vibrational coupling in enzyme catalyzed reactions. FEBS Lett. 589, 2200-2206.

(13) Chen, C.-C., Lin, Y.-C., Sheng, W.-H., Chen, Y.-C., Chang, S.C., Hsia, K.-C., Liao, M.-H., and Li, S.-Y. (2011) Genome sequence of a dominant, multidrug-resistant acinetobacter baumannii strain, tcdcab0715. J. Bacteriol. 193, 2361-2362.

(14) Ayala-del-Rio, H. L., Chain, P. S., Grzymski, J. J., Ponder, M. A., Ivanova, N., Bergholz, P. W., Di Bartolo, G., Hauser, L., Land, M., Bakermans, C., Rodrigues, D., Klappenbach, J., Zarka, D., Larimer, F., Richardson, P., Murray, A., Thomashow, M., and Tiedje, J. M. (2010) The genome sequence of psychrobacter arcticus 273-4, a psychroactive siberian permafrost bacterium, reveals mechanisms for adaptation to low-temperature growth. Appl. Environ. Microbiol. 76, 2304-2312.

(15) Bergmeyer, H. U., Gawehn, K., Klotzsch, H., Krebs, H. A., and Williamson, D. H. (1967) Purification and properties of crystalline 3hydroxybutyrate dehydrogenase from rhodopseudomonas spheroides. Biochem. J. 102, 423-431.

(16) Persson, B., and Kallberg, Y. (2013) Classification and nomenclature of the superfamily of short-chain dehydrogenases/ reductases (sdrs). Chem.-Biol. Interact. 202, 111-115.

(17) Kavanagh, K. L., Jornvall, H., Persson, B., and Oppermann, U. (2008) Medium- and short-chain dehydrogenase/reductase gene and protein families: The sdr superfamily: Functional and structural diversity within a family of metabolic and regulatory enzymes. Cell. Mol. Life Sci. 65, 3895-3906.

(18) Tokiwa, Y., and Ugwu, C. U. (2007) Biotechnological production of (r)-3-hydroxybutyric acid monomer. J. Biotechnol. 132, 264-272.

(19) Ren, Q., Ruth, K., Thony-Meyer, L., and Zinn, M. (2010) Enatiomerically pure hydroxycarboxylic acids: Current approaches and future perspectives. Appl. Microbiol. Biotechnol. 87, 41-52.

(20) Jones, H. B. L., Crean, R. M., Matthews, C., Troya, A. B., Danson, M. J., Bull, S. D., Arcus, V. L., van der Kamp, M. W., and Pudney, C. R. (2018) Uncovering the relationship between the change in heat capacity for enzyme catalysis and vibrational frequency through isotope effect studies. ACS Catal. 8, 5340-5349.

(21) Gadda, G., and Sobrado, P. (2018) Kinetic solvent viscosity effects as probes for studying the mechanisms of enzyme action. Biochemistry 57, 3445-3453.

(22) Brouwer, A. C., and Kirsch, J. F. (1982) Investigation of diffusion-limited rates of chymotrypsin reactions by viscosity variation. Biochemistry 21, 1302-1307.

(23) Blacklow, S. C., Raines, R. T., Lim, W. A., Zamore, P. D., and Knowles, J. R. (1988) Triosephosphate isomerase catalysis is diffusion controlled. Appendix: Analysis of triose phosphate equilibria in aqueous solution by $31 \mathrm{p} \mathrm{nmr.} \mathrm{Biochemistry} \mathrm{27,} \mathrm{1158-1167.}$

(24) Johnson, K. A. (1992) Transient-state kinetic analysis of enzyme reaction pathways. In The Enzymes (Sigman, D. S., Ed.) pp 1-61, Academic Press.

(25) Hartley, B. S., and Kilby, B. A. (1954) The reaction of pnitrophenyl esters with chymotrypsin and insulin. Biochem. J. 56, 288-297. 
(26) Weber, J. P., and Fink, A. L. (1980) Temperature-dependent change in the rate-limiting step of beta-glucosidase catalysis. J. Biol. Chem. 255, 9030-9032.

(27) Patel, M. P., Liu, W. S., West, J., Tew, D., Meek, T. D., and Thrall, S. H. (2005) Kinetic and chemical mechanisms of the fabgencoded streptococcus pneumoniae beta-ketoacyl-acp reductase. Biochemistry 44, 16753-16765.

(28) Silva, R. G., de Carvalho, L. P., Blanchard, J. S., Santos, D. S., and Basso, L. A. (2006) Mycobacterium tuberculosis beta-ketoacylacyl carrier protein (acp) reductase: Kinetic and chemical mechanisms. Biochemistry 45, 13064-13073.

(29) Wolfenden, R. (2014) Massive thermal acceleration of the emergence of primordial chemistry, the incidence of spontaneous mutation, and the evolution of enzymes. J. Biol. Chem. 289, 3019830204.

(30) Wolfenden, R. (2014) Primordial chemistry and enzyme evolution in a hot environment. Cell. Mol. Life Sci. 71, 2909-2915. 